Case Report

\title{
46, XXovotesticular disorder of sex development with serous cystadenomas of the ovary
}

\author{
Gwendolyn F. ${ }^{1}$, Munde S. ${ }^{2}$, Patwardhan P.P. ${ }^{3}$, Naik L. ${ }^{4}$ \\ ${ }^{1}$ Dr. Fernandes Gwendolyn, Associate Professor, ${ }^{2}$ Dr. Shital Munde, Fellow in Uropathology, ${ }^{3}$ Dr. Pranav \\ Pramod Patwardhan, Final year Resident, above authors are affiliated with Department of Pathology, Seth G. S. \\ Medical College \& K.E.M. Hospital, Mumbai, ${ }^{4}$ Dr. Leena Naik, Professor and Head of Department of \\ Pathology, Lokmanya Tilak Municipal Medical College \& Hospital, Mumbai, Maharashtra, India.
}

Address for Correspondence: Dr. Fernandes Gwendolyn, Email: drgwenfern@yahoo.co.in

\begin{abstract}
Ovotesticular disorder of sex development (DSD) is an extremely rare condition and is defined as presence of both ovarian and testicular tissue in the same individual, regardless of the karyotype and the status of the external or internal genitalia. This disorder was formerly called True hermaphroditism and 400-500 cases have been reported in literature till date. Various chromosomal abnormalities have been described but 46XX is the commonest. Both Mullerian and Wolffian duct derivatives are present in the same individual and the external as well as internal genitalia display a spectrum of phenotypes. The external genitalia are ambiguous and a uterus and a phallus areoften present. The gonads may be ovotestis or a combination of ovary on one side and testes or ovotestis on the other side. These gonads are prone to develop both benign and malignant neoplasms. Tumors like dysgerminoms, seminomas, gonadoblastoma, and mucinous cystadenomas have been described. Serous cystadenomas however, have not been described.
\end{abstract}

We describe a case of 46, XXovotesticular DSD with multiple serous cystadenomas of the left gonad which was exclusively ovary, while the right gonad was ovotestis.

Keywords: Ovotesticular DSD; ambiguous genitalia; neoplasm; cystadenoma, serous

\section{Introduction}

Ovotesticular disorder of sex development (DSD) is an unusual condition and is characterized by presence of both ovarian and testicular tissue in the same individual, regardless of the karyo type and status of external or internal genitalia [1].

It was formerly called True Hermaphroditism and 400-500 cases have been reported in literature[2,3]. Various karyo types have been described but 46XX is the commonest [4].

Both Mullerian and Wolffian duct derivatives are present in the same individual. The external genitalia are ambiguous, and both the external and internal genitalia display a spectrum of phenotypes.
The gonads may be ovotestis or a combination of ovary on one side and testes or ovotestis on the other side.

These patients are prone to develop neoplasms of the gonads and a variety of tumors like dysgerminomas, seminomas, gonadoblastoma, and mucinous cystadenomas have been described $[2,5]$.

Serous cystadenomas have not been described. We describe an unusual case of 46, XX ovotesticular DSD with multiple serous cystadenomas of the left gonad which was exclusively ovary, while the right gonad was ovotestis.

Manuscript received: $6^{\text {th }}$ November 2017

Reviewed: $16^{\text {th }}$ November 2017

Author Corrected: $24^{\text {th }}$ November 2017

Accepted for Publication: 30 $0^{\text {th }}$ November 2017 


\section{Case Report}

\section{Case Report}

A 20- year- old individual who was reared as a male, presented two years ago with abdominal pain and cyclical bleeding through an opening below a phallus-like structure. These episodes of pain and bleeding and bilateral gynecomastia occurred in the past 5 years causing him to seek medical attention. Ambiguous genitalia were noted by parents at birth but no medical assistance was taken.

On examination, well developed breasts and pubic hair of adult sexual maturity with a rating of P5 B5 (Stage Vadult) was seen (Fig 1). Ambiguous external genitalia with a $4 \mathrm{~cm}$ phallus and hypospadias were also seen. There was a single perineal opening below the phallus, but no palpable external gonads.

The Hormonal profile showed the following: Follicle stimulating hormone $5.8 \mathrm{mIU} / \mathrm{ml}$, Leutinizing hormone $3.08 \mathrm{mIU} / \mathrm{ml}$, and testosterone $0.39 \mathrm{ng} / \mathrm{ml}$, all of which were within normal limits. DHEAS (Dehydroepiandrosteronesulphate) level was $2.52 \mathrm{ug} / \mathrm{dl}$ which was markedly decreased from a normal of 65-380 ug/dl.

Magnetic resonance imaging studies showed an abnormal small sized uterus with vagina, bilateral fallopian tubes and ovaries. Micturating cystourethrography showed a normal capacity bladder. Retrograde urethrography could not be done as patient had no urethral opening in the phallus.

A karyotyping study was done and the patient showed 46, XX karyotype. The patient was counselled but was lost to follow-up.

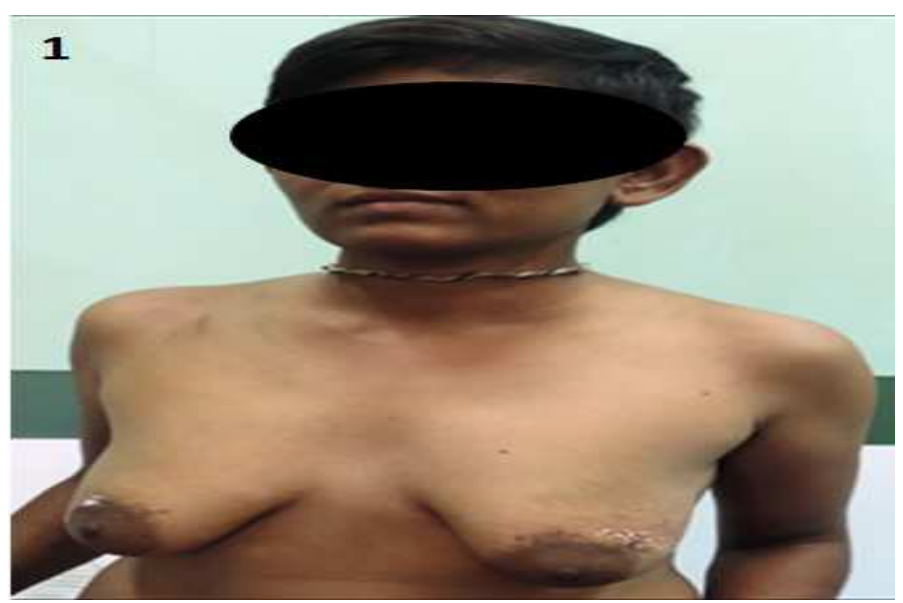

Fig 1: Bilateral gynaecomastia

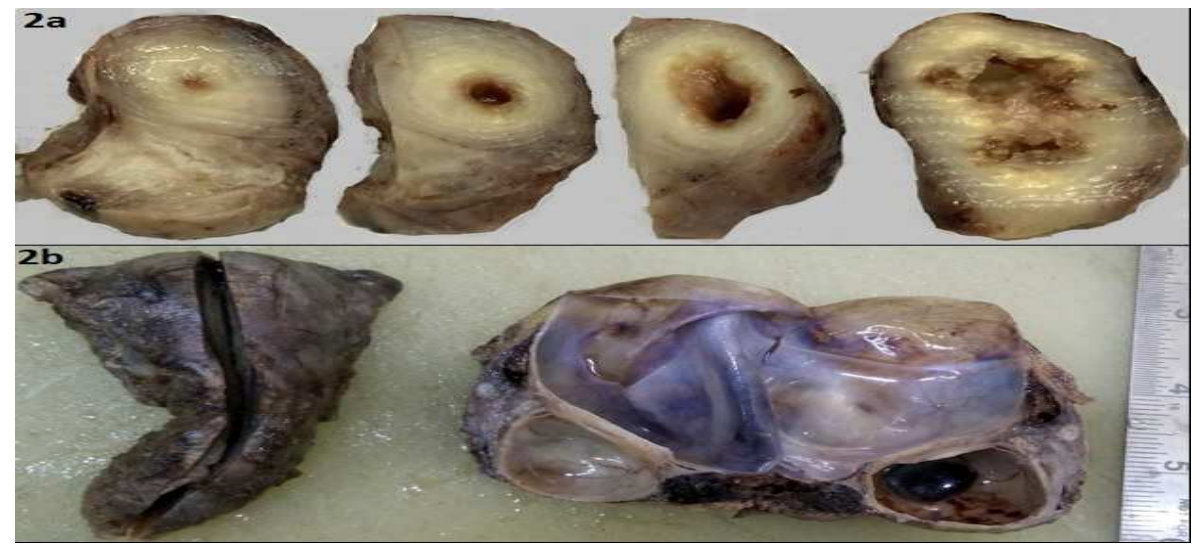

Figure: (2.) 2a. Right tubo-ovarian mass: Single thick walled tubular structure, $6 \mathrm{~cm}$ in length. Lumen of varying diameter, filled with necrotic material. $2 b$.Uterus with cervix and stumps of fallopian tubes; Left ovary enlarged \& cystic; cut surface- 2 cysts measuring $6 \times 6 \times 4 \mathrm{~cm}$ and $3 \times 3 \times 2 \mathrm{~cm}$ with smooth wall 
Case Report

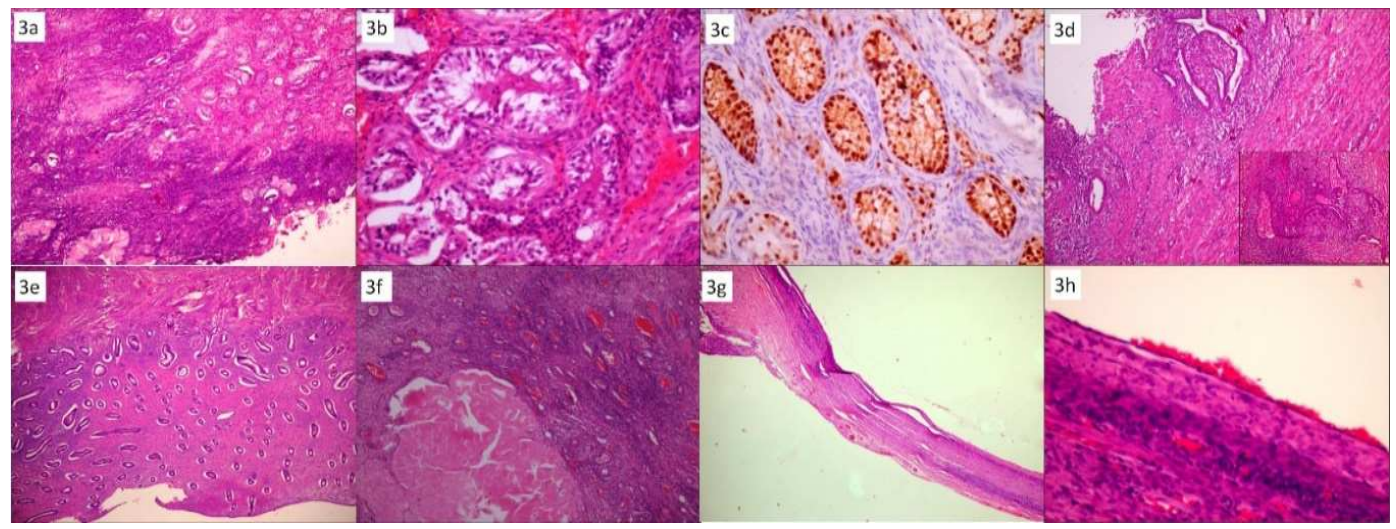

Fig 3: (3.)a. Right ovotestis: ovarian stroma with corpus albicans and testis with immature seminiferous tubules \& Leydig cells (H\&E x100). 3b. Immature seminiferous tubules (H\&E x400). 3c. ImmunohistochemistryCalretinin highlighting Sertoli cells and Leydig cells (x400). 3d. Salpingitis: Blunted, shortened plicae surrounded by chronic inflammatory cells (Inset-Fused plicae producing a pseudoglandular pattern) (H\&E $\mathrm{x} 100)$. 3e. Uterus showing proliferative endometrium (H\&E x40). 3f. Left ovary with ovarian stroma and corpus albicans. No testicular tissue seen. (H\&E x100). 3g.Fibrous cyst wall lined by a single layer of cuboidal cells (H\&E x100). 3h. High power of cyst wall lined by columnar epithelium with bland nuclear features. (H\&E $\mathrm{x} 400)$.

A year later, the patient presented with acute abdomen in our emergency services. Ultrasonography revealed right ovarian torsion while the left ovary showed a hemorrhagic cyst. An emergency laparotomy with right salpingo-oophorectomy was done and the specimen sent for the histopathological examination.

We received a right salpingo-oophorectomy specimen which showed a tubulo-ovarian mass measuring $6 \mathrm{~cm}$ in length and showing varying diameter from 1 to $2.5 \mathrm{cms}$. This tubulo-cystic structure was filled with necrotic material and showed few yellow areas in its wall. Histopathology revealed a structure of a fallopian tube showing acute on chronic salping it is with microabscesses and classical ovotestis. The ovotestis was composed of ovarian stroma with abundant corpus albicans and testis with seminiferous tubules at one end. The seminiferous tubules were lined by both spermatogonia and Sertoli cells while Leydig cells were seen around the seminiferous tubules. Immunohistochemistry showed strong Calretininpositivity for Sertoli cells and Leydig cells confirming the testes.

The patient continued having pain in the abdomen for six months after surgery and a hysterectomy with left salpingo-oophorectomy was done and sent for histopathology examination. The uterus with cervix was of normal size, with an endomyometrium of $1.5 \mathrm{~cm}$ thickness. The cervix showed Nabothian cysts. A stump of the left fallopian tube was identified which was normal. The left ovary was replaced by two cysts measuring 6x6x4 $\mathrm{cm}$ and $3 \times 3 \times 2 \mathrm{~cm}$. The cysts had smooth walls with pale reddish, watery fluid within. On microscopy, the uterus showed proliferative endometrium while the left gonadshowed exclusively ovarian tissue composed of ovarian stroma and corpus albicans. Both the cysts were composed of fibrocollagenous tissue and were lined by benign columnar epithelium. No testicular tissue was seen. Thus, the patient had right- sided ovotestis and left-sided ovary with serous cystadenomas. A final diagnosis of 46, XXovotesticular DSD with right sided ovotestis and serous cystadenomas of the left ovary was made.

\section{Discussion}

Genital ambiguity is seen in 1 in 4500 births [2].The term DSD issued to define congenital conditions in which disharmony exists in between chromosomal, hormonal and anatomical sex [6]. Ovotesticular DSD refers to the presence of both ovarian and testicular tissue in the same individual, regardless of karyotype and the status of external or internal genitalia [1]. Ovotesticular DSD occurs in fewer than $10 \%$ of all DSD and 400-500 cases approximately have been reported in the literature $[2,3]$. This disorder was formerly called as true hermaphroditism but now the term ovotesticular DSD is used. In ovotesticular DSD, both Mullerian and Wolffianductderivatives are present in the 


\section{Case Report}

same individual and the genitalia are of ambiguousnature [2]. A variety of phenotypes is seen of both external and internal genitalia. Most of these patients have a well- formed uterus present [2]. The gonads may be ovotestis or a combination of ovary on one side and testis on the other. These patients are usually reared as males because of the size of the phallus and labioscrotal fusion which is of varying degrees. At puberty, feminization and menstruation occurs. Most individuals are infertile, but ovulation and spermatogenesis are possible.

Various chromosomal abnormalities have been described in these individuals and include 46, $X X$, 46, XY, 46, XX/46, XY, 47, XXY, 47, XYY, 45X, and $46, X X / 47, X X Y$ mosaicism. $90 \%$ of the patients have $46 \mathrm{XX}$ karyotype as was seen in our case. Rarely, 46 XY/46 XX mosaicism can occur [4].

These gonads are prone to develop neoplasms, both benign and malignant and the incidence of malignant tumors developing in ovotesticular DSD is $2.6 \%$. Various tumors like dysgerminoma, seminoma, gonadoblastomas, yolk sac carcinomas and mucinous cystadenomas have been described and the commonest tumors which develop are dysgerminomas [2,5,7]. Serous cystadenomas developing in such patients with ovotesticular DSD has not been described in the literature and to the best of our knowledge; this is the first case of serous cystadenomas developing in ovotesticular DSD. Conservative gonadal surgery, hormonal replacement and counselling are the mainstay of treatment in ovotesticular DSD.

\section{Conclusion}

Early and accurate diagnosis of DSD is essential as such patients have physical, psychological, and infertility issues besides having a high risk of developing malignancies. All efforts should be made to assign an appropriate gender to the patient at the earliest so as to help the individual to lead a normal life.
Ovotesicular DSD is associated with a spectrum of both benign and malignant neoplasms, most common tumor being dysgerminoma. This case has been published because to the best of our knowledge, this is the only reported case of serous cystadenomas occurring in a case of 46, XX ovotesticular DSD.

\section{Funding: Nil, Conflict of interest: None Permission of IRB: Yes}

\section{References}

1. Hisamatsu E, Nakagawa Y, Sugita Y. Two cases of late-diagnosed ovotesticular disorder of sex development. APSP J Case Rep. 2013 Oct 22;4(3): 40. eCollection 2013.

2. SwainS, Pradhan L, Satpathy R, Mahapatra P. A rare case report on ovotesticular disorders of sex development (DSD) 46XY variant. Int J Reprod ContraceptObstet Gynecol. 2014 Dec;3(4):1163-6.

3.GhofraniM.Truehermaphroditism.pathologyoutli nes.com.website.http://www.pathologyoutlines.co $\mathrm{m} /$ topic/ovarynontumortruehermaph.html

4. Donahoe PK, Schnitzer JJ. Evaluation of the infant who has ambiguous genitalia, and principles of operative management. Semin Pediatr Surg. 1996 Feb;5(1):30-40.

5. Corriden TF. Ovarian neoplasms in a hermaphrodite. N Engl J Med. 1949 Feb 17;240(7):247-9.

6. Hughes IA, Houk C, Ahmed SF, Lee PA; LWPES ConsensusGroup; ESPE Consensus Group. Consensus statement on management of inter sex disorders. Arch Dis Child. 2006 Jul;91 (7): 554-63. Epub 2006 Apr 19.

7. Montero M, Méndez R, Valverde D, Fernández JL, Gómez M, Ruíz C.True hermaphroditism and normal male external genitalia: a rare presentation. Acta Paediatr. 1999 Aug;88(8):909-11.

\section{How to cite this article?}

Gwendolyn F, Munde S, Patwardhan P.P, Naik L. 46, XXovotesticular disorder of sex development with serous cystadenomas of the ovary. Int J Med Res Rev 2017;5(11):952-955.doi:10.17511/ijmrr. 2017.i11.06 